[aHR]: 6.76; 95\% CI, 5.19-8.82) and administration of HAART (aHR: 1.53 ; 95\% CI, 1.28-1.84) were the factors associated with HZ. Life-table method was used to divide the duration of HIV from diagnosis into two phases, namely, $\leq 4$ years and $>4$ years. Initiation of HAART within 4 years of HIV diagnosis was associated with an increased risk of $\mathrm{HZ}$ (HR: 1.79, 95\% CI: $1.48-2.16, \mathrm{p}<0.0001)$ and after 4 years of HIV diagnosis was associated with a decreased risk of $\mathrm{HZ}$ infection (HR: 0.60, 95\% CI: 0.47-0.78, p < 0.0001). Among HIV-infected patients on HAART, with $\geq 85 \%$ adherence was showed significantly lower risk of developing HZ (HR: 0.40, 95\% CI: 0.19-0.85, $\mathrm{p}<0.001)$.

Conclusion With high level of HAART adherence, it had significantly lowered HZ infection risk. Therefore, we suggested emphasising the importance of early treatment and HAART adherence.

\section{P16.26 HUMAN IMMUNODEFICIENCY VIRUS (HIV) - MYCOBACTERIUM TUBERCULOSIS (TB) CO-INFECTION IN SRI LANKA}

Dpcka Lal*. National Hospital of Sri Lanka

\subsection{6/sextrans-2015-052270.573}

Background TB and HIV co-infection is considered to occur worldwide. Immunosuppression by HIV makes patients vulnerable to be infected with $\mathrm{TB}$ and they are more prone to get severe disease. Prevalence of TB and HIV in Sri Lanka is $4.2 \%$ and $<0.1 \%$ respectively. Relationship between HIV and TB is not yet clearly defined in Sri Lanka. The objective of this preliminary study is to describe the epidemiology of HIV - TB coinfection in Sri Lanka.

Methods 54 sexually active patients with histopathologically or microbiologically proven Tuberculosis were screened for HIV with ELISA antibody test. Positive ELISA was confirmed by western blot test.

Results Patients were 17 to 54 years of age. Male: Female = 33:21. 38 and 16 patients had pulmonary and extra pulmonary TB respectively. Only 02 male patients had positive ELISA test for HIV but both were negative for western blot test.

Conclusion HIV - TB co-infection is not a significant occurrence in Sri Lanka yet. There for HIV should not be considered as an important predisposing factor for TB in Sri Lanka and It is not rational to screen all TB patients for HIV as it is not cost effective for a resource poor country.

Disclosure of interest Nothing to disclose.

\section{P16.27 FRAMINGHAM CHD AND CVDS RISK EQUATIONS IN HIV AND HIV/HCV POPULATION: A COMPARISON STUDY AMONG MALAYSIAN HIV INFECTED SUBJECTS ON ART}

${ }^{1} \mathrm{~N}$ Hejazi, ${ }^{1} \mathrm{R}$ Rajikan, ${ }^{2} \mathrm{CLK}$ Choong. ${ }^{1}$ National University of Malaysia; ${ }^{2}$ Sungai Buloh Hospital

\subsection{6/sextrans-2015-052270.574}

Introduction The possible underestimated risks of coronary heart disease (CHD) and cardiovascular diseases (CVDs) calculated by the Framingham Risk Score (FRS) models were reported for subjects with HIV and hepatitis $\mathrm{C}$ virus (HCV) infection. This novel study aimed to compare the CHD and CVDs risk factors as well CHD and CVDs events predictions using FRS between HIV- infected and HIV/HCV subjects on antiretroviral therapy (ART) in Malaysia.

Methods This retrospective study was conducted with a purposive sampling of 2046 HIV patients on ART in an outpatient clinic in Malaysia age, gender, lipid profile, blood pressure, smoking, diabetes status, immunity indices, and ART using digital medical records. Risks were predicted using FRS models (2002) for CHD and FRS formula (2008) for CVDs. Descriptive, independent sample T-test and Chi-square statistical tests were applied.

Results CHD and CVD risks were estimated in 1850 subjects (median age 46 years, 20\% female) only with HIV and 196 of HIV/HCV subjects (median age 43 years, $4 \%$ female). The HIV/ HCV group had significant lower mean levels of total cholesterol, HDL, LDL, triglyceride and systolic blood pressure while mean age was significant higher in HIV group $(\mathrm{p}<0.05)$. HCV/ HIV group had significant proportion of Tenofovir receivers $(34.6 \%$ vs $16.6 \%)$ and lower CD4 count level ( $p<0.05)$. The intermediate and high risks of CHD were prevalent among HIV/ HCV and HIV subjects as $6.1 \%$ vs $6.6 \%$ and $3.1 \%$ vs $3.6 \%$ respectively. Also HIV vs $\mathrm{HIV} / \mathrm{HCV}$ subjects had intermediate CVD risks as $40.9 \%$ vs $38.6 \%$ while $8.3 \%$ vs $8.2 \%$ had high risk CVDs. Points and risks percentages of CVDs and CHD were not significant different between $\mathrm{HIV}$ and $\mathrm{HIV} / \mathrm{HCV}$ groups.

Conclusion Risk of CHD and CVDs were similar in HIV and HIV/HCV groups. This Study suggests a need for more specific FRS equations since the serum lipid profiles influence by some factors such as immunity status, ART and HCV rather than traditional risks.

Disclosure of interest statement There is no conflict of interest.

\section{P16.28 FRAMINGHAM CORONARY HEART DISEASE AND CARDIOVASCULAR RISK ASSESSMENTS OF HIVIAIDS MALAYSIAN POPULATION ON HAART: THE IMPORTANCE OF RISKS EVALUATIONS AND PREDICTIONS}

${ }^{1} \mathrm{~N}$ Hejazi, ${ }^{1} \mathrm{R}$ Rajikan, ${ }^{2} \mathrm{CLK}$ Choong. 'National University of Malaysia; ${ }^{2}$ Sungai Buloh Hospital

\subsection{6/sextrans-2015-052270.575}

Background Coronary heart disease (CHD) and cardiovascular diseases (CVDs) events have increasing trends mainly due to the multiple and complex mechanisms of chronic inflammation and anti-retroviral drugs adverse effects during HIV course. Due to the lack of information this study aimed to analyse the CHD and CVDs risk profiles, estimate the probability of events and evaluate the accuracy of the Framingham CHD equations comprehensively in HIV-infected Malaysian subjects on highly active antiretroviral therapy (HAART).

Methods This is a cross-sectional study with a purposive sampling of 2046 HIV patients on HAART in an outpatient infectious disease clinic in Selangor Malaysia. Using digital medical records. all variables for Framingham equations including demographics, gender, fasting plasma glucose and lipid profiles, blood pressure, smoking and diabetes status, hypertension treatment, immunity indices and antiretroviral therapy were collected. 10years CHD risks were predicted using Framingham Risk Score (FRS1998 and FRS 2002) models while CVDs risk by specific FRS (2008). Data analyses included descriptive statistics and binary logistic regression. 
Results CHD risks were estimated in 1920 (FRS 1998) and 2046 (FRS 2002) of HIV subjects while CVDs risk were evaluated in 1927 subjects. Median ages and percentage of male were 44 years and 81.4\% (FRS 1998, 2002 and FRS 2008). Approximatly 90\% were receiving NNRTI+ NRTI and just 13\% and $10 \%$ were taking tenofovir and a protease inhibitor. Beside $11 \%$ were smokers while $14 \%$ as diabetic. Dyslipidemia was seen at least in $45 \%$ of population. Equally $8 \%$ had CD4 count $<200$ cells $/ \mathrm{mm}^{3}$ and 9\% hepatitis C. Importantly $10 \%$ and $4 \%$ had intermediate and high CHD risks (FRS 1998) and 6.6\% and $3.3 \%$ with intermediate and high risks for CHD based on FRS (2002) intermediate and high risk of CVDs was prevalent in while $39 \%$ and $8 \%$ of HIV subjects. Among all studied variables, higher total cholesterol levels and older age were the strong risk predictors for CHD and CVDs $(\mathrm{p}<0.05)$.

Conclusions We found a high prevalence of dyslipidemia while the CHD risks measured by the Framingham scales 1998 and 2002 were low. Notably CVDs risk was high thus further investigations as well preventive management should be prioritised in this population.

\section{P16.29 PREVALENCE OF METABOLIC RISK FACTORS IN HIV- INFECTED POPULATION UNDER ANTIRETROVIRAL THERAPY IN NORTHERN MALAWI}

${ }^{1} \mathrm{CC}$ Chung ${ }^{*},{ }^{1} \mathrm{HH}$ Lin, ${ }^{2} \mathrm{CC}$ Mtika, ${ }^{3} \mathrm{~J}$ Wu. ${ }^{1}$ National Taiwan University, Institute of Epidemiology and Preventive Medicine; ${ }^{2}$ Mzuzu Central Hospital (Malawi); ${ }^{3}$ Luke International in Malawi

\subsection{6/sextrans-2015-052270.576}

Introduction With increased availability of antiretroviral therapy (ART), the life expectancy of people with human immunodeficiency virus (HIV) infection is expected to improve substantially. On the other hand, life-long ART may be associated with increased obesity, diabetes, and hypertension. The situation of obesity, diabetes, and hypertension in HIV-infected population in Malawi is unclear.

Methods We conducted a cross-sectional study including adult HIV-infected patients under ART treatment in a teaching hospital from northern Malawi. Trained field workers performed anthropometric measurements, blood pressure exam, and finger prick test for blood glucose. We followed the WHO and International Diabetes Federation (IDF) criteria to define obesity, hypertension, and diabetes. We compared the prevalence of metabolic risk factors in our study population to that in the 2009 STEPwise approach to Surveillance (STEPS) survey in Malawi.

Results The study included 410 participants (20.97\% males). The prevalence of overweight $(25 \leq \mathrm{BMI}<30)$ and obesity (BMI $\geq 30$ ) was $22.9 \%$ (95\% CI: $18.9-26.9 \%$ ) and $10.0 \%$ (95\% CI: 7.1-12.9\%), respectively. The prevalence was higher than that in the STEPS survey (21.9\% for overweight and $4.6 \%$ for obesity). Notably the situation of overweight and obesity was much worse in females $(26.8 \%$ and $12.3 \%)$ than in males $(8.2 \%$ and $1.2 \%)$. Old age (OR: 1.064 per year, $\mathrm{p}=0.0016)$ and alcohol drinking (OR: 7.309, $\mathrm{p}=0.0005$ ) were significant associated with obesity. The prevalence of diabetes or impaired fasting glucose (20.2\%, 95\% CI: 16.3-24.1\%) was higher than that in STEPS survey $(9.8 \%)$. The overall prevalence of hypertension was $18.1 \%$ (95\% CI: $14.3-21.8 \%$ ), lower than that in the STEPS survey (32.9\%).

Conclusion Our study provides an initial assessment of the burden of metabolic risk factors among HIV-infected population under long-term ART treatment in Malawi. The high prevalence of metabolic risk factors in this population is concerning. Longterm impact on the burden of noncommunicable diseases warrants further investigation.

Disclosure of interest statement This study was funded by Luke international belongs Pingtung Christian hospital. And that was leaded by National Taiwan University for academic research.

\section{P16.30 EPIDEMIOLOGY OF ANAL INFECTION IN HIV INFECTED PATIENTS ATTENDING A SEXUALLY TRANSMITTED INFECTION CLINIC IN BRAZIL}

Boldrini Nat*, LP Bondi, LC Spano, LB Freitas, AE Miranda. Post-Graduation Program in Infectious Diseases, Universidade Federal Do Espirito Santo

\subsection{6/sextrans-2015-052270.577}

Introduction Anal squamous cell carcinoma is rare in the general population but certain populations, such as persons with HIV, are at increased risk. High-risk populations can be screened for anal cancer using strategies similar to those used for cervical cancer. The objective of this study was determinating the prevalence, genotype distribution and risk factors associated with anal HPV infection among persons of both sexes with HIV attending a STI clinic in Espirito Santo, Brazil.

Methods Cross sectional study assessing HIV-infected persons. A comprehensive survey was administered that included a demographic and behavioural assessment. Anal specimens were collected for cytology and HPV screening using Polymerase Chain Reaction.

Results A total of 169 patients completed the study, 122 female and 47 male, mean age was 40.3 years, $81.4 \%$ lived in Vitoria, the average education was 8.6 years, $48.8 \%$ were married, $42.1 \%$ had between 5 and 20 sexual partners, $25.4 \%$ initiated sexual activity before age $15.72 \%$ reported anal sexual activity, $58.3 \%$ had a history of STI, the most frequent being condyloma acuminata (25.4\%) followed by syphilis (9\%). 31.6\% knew to be HIV positive for more than 10 years, $65.1 \%$ had an undetectable viral load, only $3.5 \%$ had CD4 below 200 cells and $82.9 \%$ were taking HAART. Anal cytology in both sexes had a $13.2 \%$ prevalence changes. The prevalence of HPV infection of any type was $71 \%$, and high-risk HPV types were $52.4 \%$. The HPV types most frequent high-risk types were: 16, 51 and 53. 37.3\% had multiple HPV types.

Conclusions Anal HPV is common among HIV-infected persons attending this STI clinic, repeated annual cytology screening for HIV-infected, particularly for those with increased immunosuppression, anal HPV, history of the other STIs, or abnormal cervical cytology will increase the likelihood of detecting AIN 2-3.

Disclosure of interest statement There is no conflict of interest.

\section{P16.31 IMPROVING TIMELY HIV RESULTS IN KEY POPULATIONS: RAPID TEST ANTIBODY EVALUATION FOR HIV DIAGNOSIS IN GUATEMALA, 2012-2013}

${ }^{1}$ E Arana Flora, ${ }^{2} \mathrm{~L}$ Castillo-Signor, ${ }^{2} \mathrm{P}$ Marchorro, ${ }^{3} \mathrm{~V}$ Girón, ${ }^{2} \mathrm{~A}$ Lopez, ${ }^{2} \mathrm{C}$ Escobar, ${ }^{1} \mathrm{C}$ Vargas, ${ }^{1} \mathrm{R}$ Mendizabal-Burastero, ${ }^{1} \mathrm{~S}$ Morales-Miranda*. ${ }^{1} \mathrm{HIV}$ Unit, Centers for Health Studies, Del Valle University; ${ }^{2}$ National Health Laboratory, Ministry of Health, Guatemala; ${ }^{3}$ HIV National Program, Ministry of Health, Guatemala

\subsection{6/sextrans-2015-052270.578}

Introduction Guatemala has a concentrated HIV epidemic among key populations; in 2003, the first rapid test validation in Central America was performed. A national HIV testing algorithm 